\title{
Wobble base-pairing slows in vivo translation elongation in metazoans
}

\author{
MICHAEL STADLER ${ }^{1}$ and ANDREW FIRE ${ }^{1,2,3}$ \\ ${ }^{1}$ Department of Genetics, Stanford University, Stanford, California 94305-5324, USA \\ ${ }^{2}$ Department of Pathology, Stanford University, Stanford, California 94305-5324, USA
}

\begin{abstract}
In the universal genetic code, most amino acids can be encoded by multiple trinucleotide codons, and the choice among available codons can influence position-specific translation elongation rates. By using sequence-based ribosome profiling, we obtained transcriptome-wide profiles of in vivo ribosome occupancy as a function of codon identity in Caenorhabditis elegans and human cells. Particularly striking in these profiles was a universal trend of higher ribosome occupancy for codons translated via G:U wobble base-pairing compared with synonymous codons that pair with the same tRNA family using G:C base-pairing. These data support a model in which ribosomal translocation is slowed at wobble codon positions.
\end{abstract}

Keywords: ribosome; footprinting; codon usage; translation; wobble

\section{INTRODUCTION}

Though most amino acids can be encoded by multiple RNA sequences, organisms sample from this sequence space in a biased manner, with individual codons showing species-specific over- or underrepresentation (Grantham et al. 1980, 1981; Palidwor et al. 2010). One biological consequence of codon choice is to influence local translation elongation rates on messenger RNAs. The rate of translation elongation can influence both the amount and quality (e.g., folding) of protein produced (Nussinov 1981; Pedersen 1984; Varenne et al. 1984; Purvis et al. 1987; Xia 1996), indicating that the specific codon sequence, beyond merely dictating the amino acid sequence, can be an important determinant of the final biological output of a gene.

Experiments in microorganisms have demonstrated that a codon's translation rate correlates positively with both the frequency of usage and acceptor tRNA concentration, consistent with models in which tRNA selection on the ribosome is rate-limiting in elongation (Varenne et al. 1984; Sorensen et al. 1989). However, rare codons can, in some cases, be decoded rapidly and frequent codons slowly (Bonekamp et al. 1989; Curran and Yarus 1989; Nakamura and Sugiura 2011). Codons translated by identical tRNAs using different basepairing geometries can also be decoded at different rates

\footnotetext{
${ }^{3}$ Corresponding author.

E-mail afire@stanford.edu.

Article published online ahead of print. Article and publication date are at http://www.rnajournal.org/cgi/doi/10.1261/rna.02890211.
}

(Thomas et al. 1988; Curran and Yarus 1989; Kato et al. 1990). These results were derived using limited sets of codons in specific sequence contexts; a systematic examination of codon translation elongation rates examining many transcripts and diverse sequence contexts has not been previously possible.

To explicitly investigate translational effects related to the character of codon:anti-codon base-pairing interactions, we focused on pairs of codons that are (1) identical in the first two bases, (2) differ in the third base (cytosine in one, uracil in another), and (3) are translated by a shared tRNA family with a $G$ in the anti-codon wobble position (Fig. 1A; Crick 1966). These conditions, which are met for eight $\mathrm{C} / \mathrm{U}$-ending codon pairs each in Caenorhabditis elegans and human (Chan and Lowe 2008), allow us to compare different codon:anti-codon geometries under circumstances where a single tRNA family is being used for both standard (Watson:Crick; G:C) recognition and wobble (G:U) base-pairing. A second category of synonymous codon pairs (seven additional pairs in human and eight in C. elegans) is decoded by shared tRNAs derived from precursors carrying an adenine in the anti-codon wobble position. This A residue is edited to inosine (Supplemental Table S1) to produce a bifunctional tRNA population recognizing both $\mathrm{C}$-ending and $\mathrm{U}$-ending codons (I:U and I:C base-pairing at the wobble position). Of the base-pairs relevant to this analysis, G:C forms the highest affinity interaction, with three hydrogen bonds, and I:C forms with a similar geometry but only two hydrogen bonds. G:U 


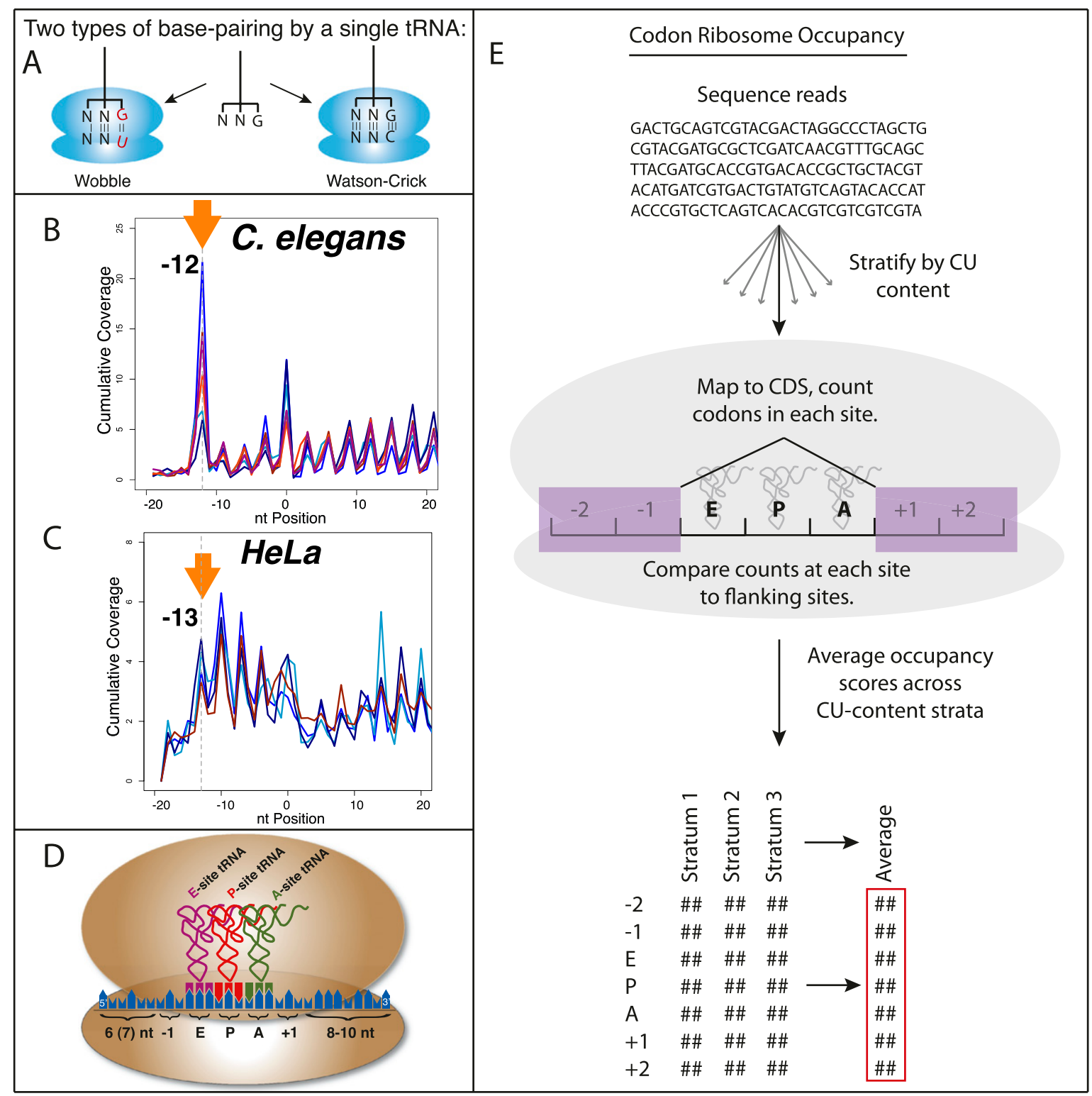

FIGURE 1. Positioning ribosome sites and analysis overview. (A) A single tRNA family with $\mathrm{G}$ in the wobble position can use two types of basepairing to decode synonymous codons. $(B, C)$ Cumulative coverage of RPF start nucleotides mapping near the initiation codon. Data shown is the aggregate normalized (per transcript) coverage across all mRNAs in $C$. elegans $(B)$ and HeLa $(C)$ data sets. Individual colored lines represent independent biological and technical replicates. Arrows indicate the peak inferred to represent the position of RPFs with the AUG in the P-site. $(D)$ Location of tRNA binding-sites and structure of RPFs inferred from data in $A$ and $B$. (E) Overview of analytical approach for determining ribosome occupancy for codon-ribosomal site combinations.

and I:U form with a less-favorable geometry and two hydrogen bonds.

Ingolia et al. (2009) recently developed a high-throughput ribosome profiling technique that utilizes the positions of mRNA fragments protected from nuclease digestion by bound ribosomes (Steitz 1969) to generate a transcriptomewide map of ribosome occupancy at subcodon resolution. As these profiles represent a snapshot of the positions of ribosomes at a specific point in time, positions where ribosomes remain for longer time periods will have a higher probability of being captured by sequencing. The rate at which a codon is translated should thus vary inversely with the occupancy of elongating ribosomes at mRNA positions featuring this codon; i.e., the presence of a slow codon should yield increased ribosome occupancy at that specific position on an mRNA. We therefore used genomewide ribosome profiling to probe the relationship between codon identity and ribosome occupancy, and investigate codon-dependent effects on ribosome elongation speed. 


\section{RESULTS}

We performed ribosome profiling (Ingolia et al. 2009) on developmentally synchronized C. elegans populations and on populations of cultured human cervical cancer cells (HeLa). As a reference and normalization for each sample, we sequenced matching alkali-sheared poly(A)-selected RNA with a similar size distribution (Ingolia et al. 2009). As expected if the recovered fragments were indeed ribosome footprints (and as observed by Ingolia), reads from these ribosome-protected fragments (RPFs) were enriched for lengths of $\sim 30-32$ nucleotides (nt), showed a strong 3-nt periodicity, and were overwhelmingly enriched in coding regions (Supplemental Fig. S1A-J). Examining cumulative RPF distributions relative to annotated initiation codons revealed a distinctive inflection point before which few RPFs are observed and after which RPFs become abundant (Fig. $1 \mathrm{~B}, \mathrm{C})$. This point is presumed to represent the location of ribosomes positioned at the initiator AUG in each message. As initiator tRNA is thought to enter at the P-site (Steitz 2008), this signal indicates a tentative location for the P-site relative to the $5^{\prime}$-nt of RPFs. This identification allows us to infer the codons occupying the putative exit (E) and aminoacyl (A) sites within each RPF (Fig. 1D). The location of this signal is consistent to within $\pm 1 \mathrm{nt}$ across a range of RPF lengths, indicating that the $5^{\prime}$-most nucleotide is a robust marker for determining ribosomal site location and that most length heterogeneity occurs at the $3^{\prime}$ end of the RPFs (Supplemental Fig. S1K-L).

In analyzing the sequence covered by RPFs from $C$. elegans and HeLa cells, we observe a combination of mild overall sequence composition biases (a preference for $U$ residues at all positions) and specific codon-associated occupancy patterns. The data yield a count of the number of ribosomes captured with each codon occupying each site within the RPF (e.g., the A-site). To control for local and compositional effects (e.g., codon usage), we normalize recovered counts for each codon in a specific ribosomal site to counts across all non-tRNA-interacting sites (for a complete description of the normalization procedure, see Methods). Higher scores-indicating the recovery of a given codon in a given site more often than expected-are indicative of an association between the presence of a given codon in a specific site and high ribosome occupancy. Codon-site combinations associated with slowed ribosomal elongation will thus be evident by higher scores in this assay.

In C. elegans, the examination of ribosome occupancy data for $\mathrm{C} / \mathrm{U}$-ending codon pairs reveals a striking pattern in the putative P-site position: For 16 of $16 \mathrm{NNC/NNU}$ codon pairs, ribosome occupancy is higher for the U-ending codon (Fig. 2A). No equivalent pattern is observed in fragmented mRNA data (Fig. 2B). This difference is significantly greater among codon pairs that are read by $\mathrm{G}: \mathrm{C} / \mathrm{G}: \mathrm{U}$ at the third position than those read by
I:C/I:U. As G:U and G:C bonds differ to a greater extent in terms of geometry and bond strength (three hydrogen bonds versus two) than I:U and I:C pairs, this result suggests that the physical characteristics of base-pairing interactions at the third codon position may influence ribosome elongation.

A distinctive wobble position-associated difference was also observed in HeLa cells (Fig. 3A). In ribosome profiling data from these human cells, we again observed an increase in ribosome occupancy associated with U-ending codons that is not observed for randomly sheared mRNA (Fig. 3B). The HeLa data differed in that the effect was robust only for codon pairs translated by tRNAs using G:C/G:U pairs at the wobble position, with a weak trend evident for I:C/I:Udecoded codons. Additionally, the G:U "slowing" signal in HeLa was strongest for the presumptive ribosomal A-site codon, distinct from the $\mathrm{P}$-site association observed in $C$. elegans data. Although the single-codon shift is potentially due to a fundamental biological difference between samples, it is certainly conceivable that this single-codon shift reflects modest differences in the isolation and preparation of ribosomes and RPFs. Specifically, it is possible that during the preparation of $C$. elegans samples, ribosomes are able to translocate one or a few codons during tissue thawing. Single-codon precision would rely on uniformly arresting translation in two very different biological samples; ruling out a single-codon difference would be difficult with tissues from distinct sources. Despite the apparent one-codon difference, the concurrence of overall wobble-position effects between C. elegans and HeLa implicates a specific effect of base-pairing interactions on ribosome elongation rate as a potentially general metazoan phenomenon.

These data suggest a model in which wobble-position interactions could serve as significant determinants of elongation rates in the two systems. To evaluate this model, we first considered a number of technical aspects of the experimental and analytical procedures that could potentially have produced a systematic bias.

First, we compared several different experimental runs from both HeLa and C. elegans to determine whether the observed ribosome retention at the wobble codons was consistently obtained. This analysis showed consistent wobble retention across two biological replicates in HeLa cells and five $C$. elegans samples derived from several larval stages (Supplemental Figs. S2A-E, S3A,B; Kim et al. 2001).

Second, we considered the possibility that drug-mediated arrest of translation during RPF preparation plays a role in the observed variation in ribosome occupancy. A series of controls performed in duplicate without cycloheximide shows an equivalent set of effects (Supplemental Figs. S2F-H, S3C-E). These results rule out a role for this drug in the observed wobble effects.

Third, we considered the possibility that a single class of mRNA might account for the consensus effects observed. To address this, we stratified the populations of mRNA by 
A
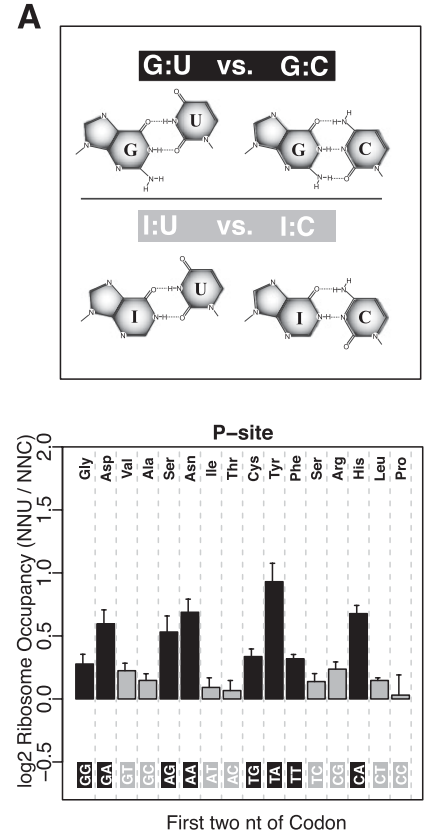

B
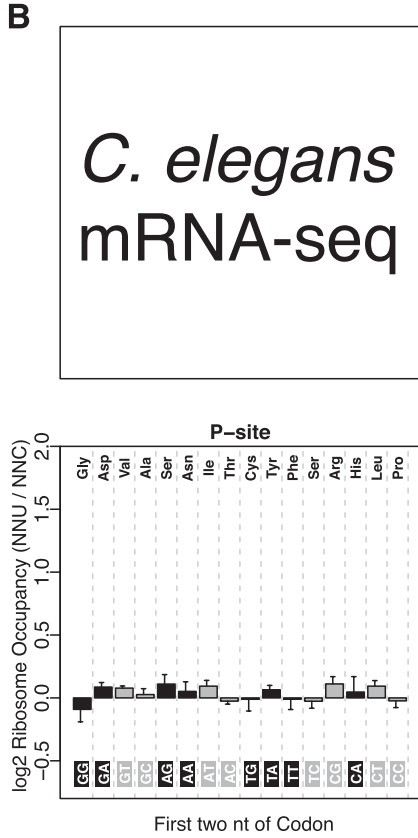

Minus-2

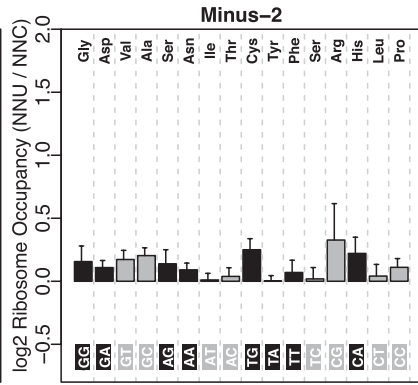

First two nt of Codon

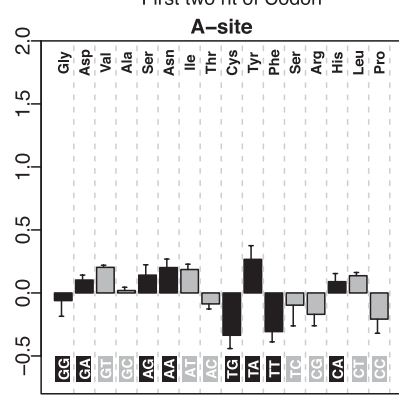

Minus-2

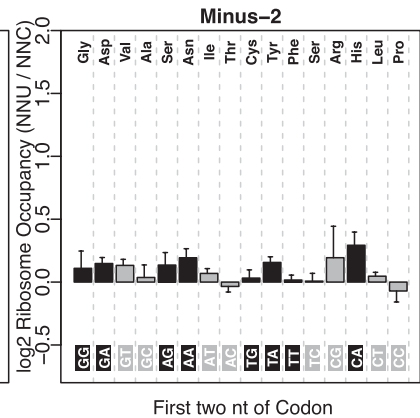

A-site

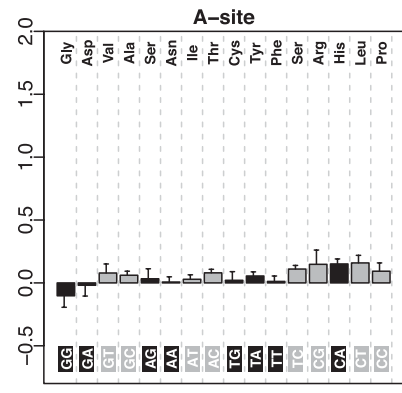

Minus-1
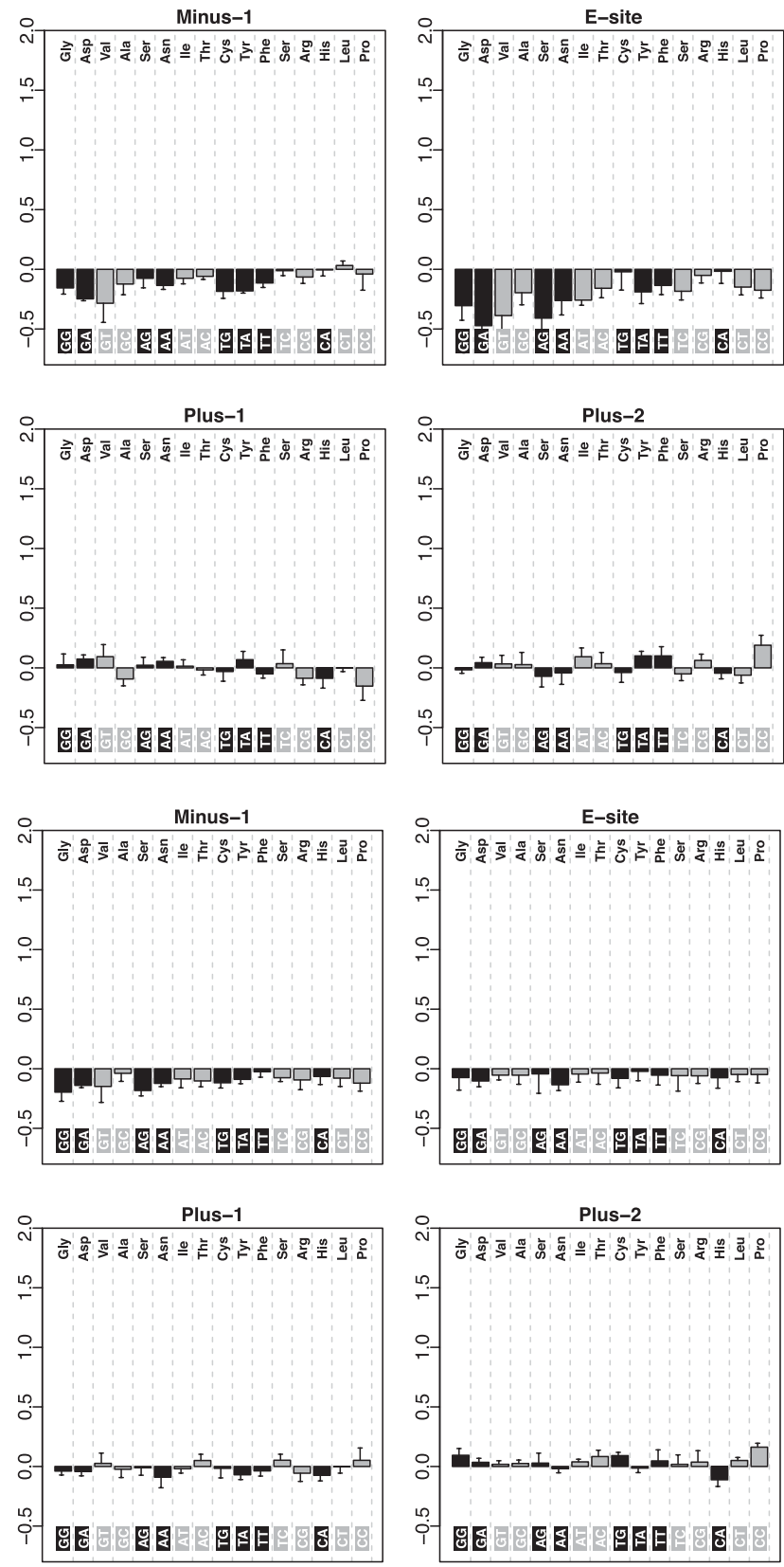

FIGURE 2. Ribosome occupancy for C/U-ending codon pairs in C. elegans (A) Wobble-position base-pairing geometries used by C/U-ending codons are shown in the top left panel. Remaining panels show the difference in ribosome occupancy for synonymous $\mathrm{C} / \mathrm{U}$-ending codon pairs (NNU / NNC). Codons that are read by G:C/G:U base-pairing at the wobble position are shaded black; pairs read by I:C/I:U are shaded gray. Error bars, SD. (B) Identical figure showing control occupancy data for alkali-sheared mRNA.

several different criteria into distinct classes. The classification of mRNA by length (Supplemental Figs. S2I-K, S3FH) and expression levels (Supplemental Figs. S2L-N, S3I$\mathrm{K})$ yielded wobble-position characteristics similar to those observed with the entire mRNA population in each class examined.

Fourth, it has been reported in some systems that ribosome occupancy (Ingolia et al. 2009) and codon usage (Tuller et al. 2010) are altered at the beginning of coding sequences. Correlations between ribosome occupancy and codon usage along the length of mRNAs could account for observed occupancy patterns for wobble codons. To address this possibility, we separated mRNA sequences into early (first 50 codons in each mRNA), late (last 50 codons in each mRNA), and middle (remaining codons) groups. This experiment revealed consistent wobble-position effects for each mRNA region (Figs. 4A-C, 5A-C). These analyses implicate the observed wobble-position effect as a consistent 
A
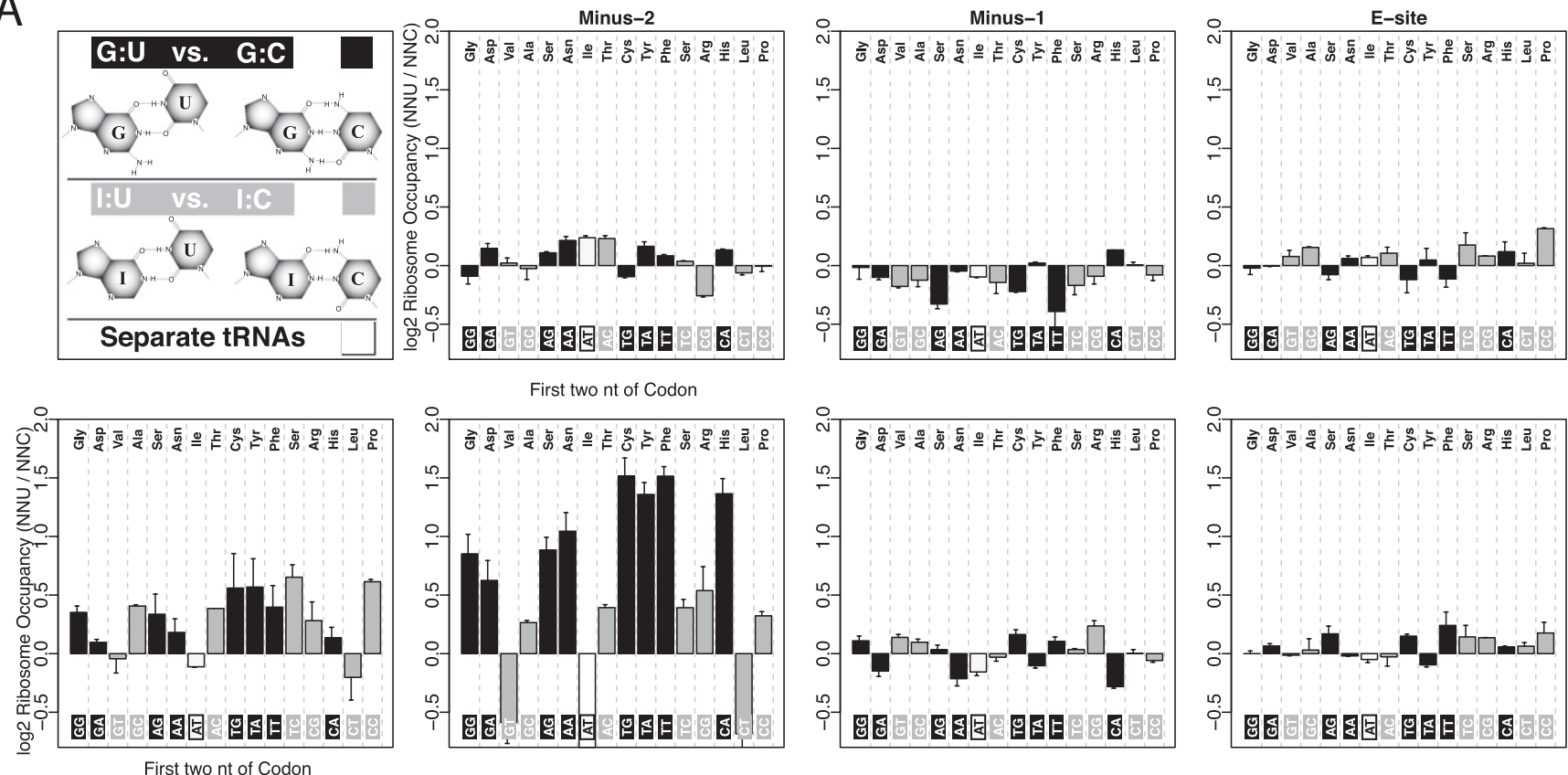

B
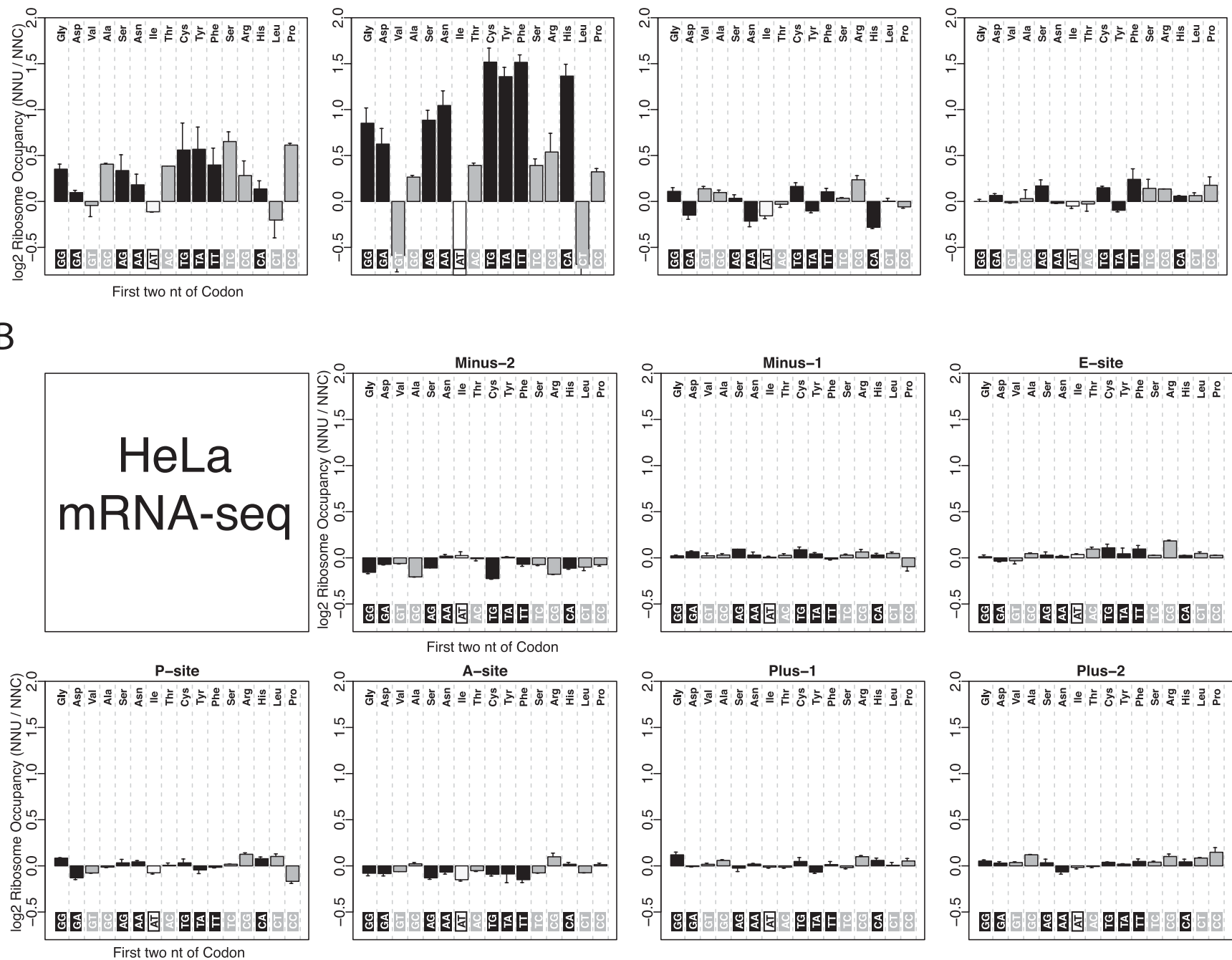

FIGURE 3. Ribosome occupancy for C/U-ending codon pairs in HeLa cells. Panels are as in Figure 2. White bar indicates that for this codon pair, the human genome encodes multiple copies of cognate tRNAs with anti-codons with A and $\mathrm{G}$ in the wobble position; codons may therefore not share a common tRNA pool. $(A)$ Data from RPF libraries; $(B)$ data for sheared mRNA.

modulator at different stages of elongation and that it is not a result of position-specific biases in codon usage.

Fifth, we considered that wobble base-pairing could change the nuclease sensitivity of ribosome-mRNA complexes. Because we filter sequence reads by size and reading frame to examine only the highest-quality RPFs, it is possible that changes in nuclease sensitivity that alter the size or reading frame of RPFs could cause reads to be excluded by our filters. To address this possibility, we repeated the analysis using all data-without filtering for reading frame or RPF length (Supplemental Figs. S4A, S5A) - and also repeated the analysis using only reads starting in either underrepresented reading frame (Supplemental Figs. S4B,C, S5B,C). In both cases, wobble-position ribosome occupancy 

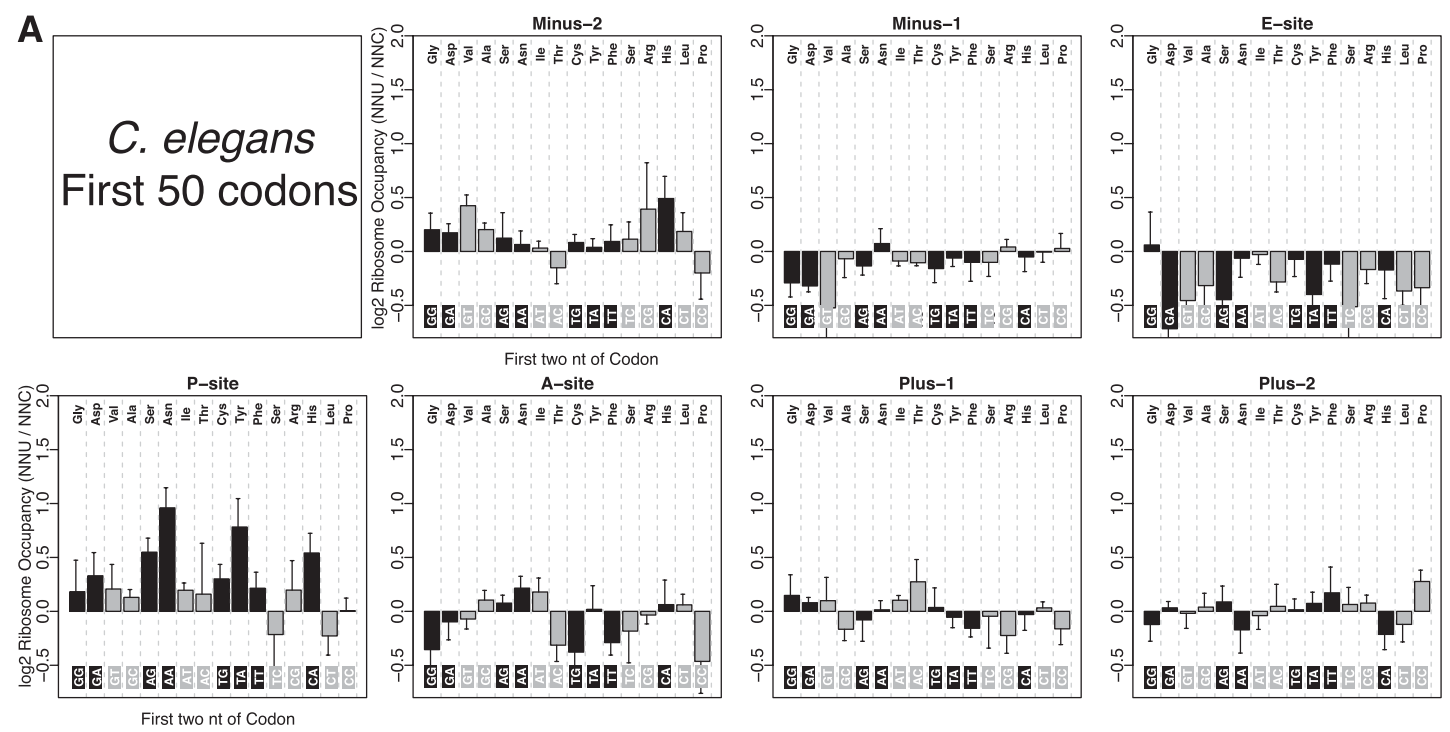

B
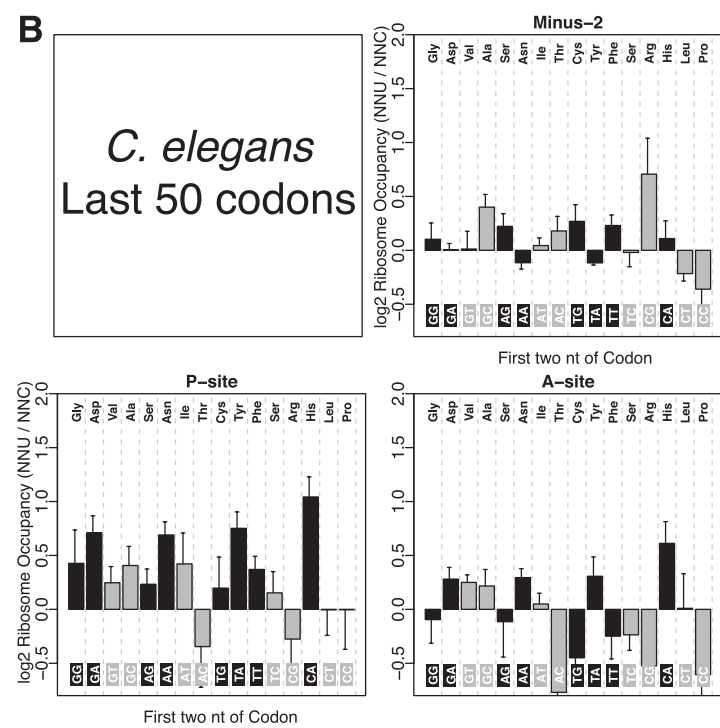

First two nt of Codon
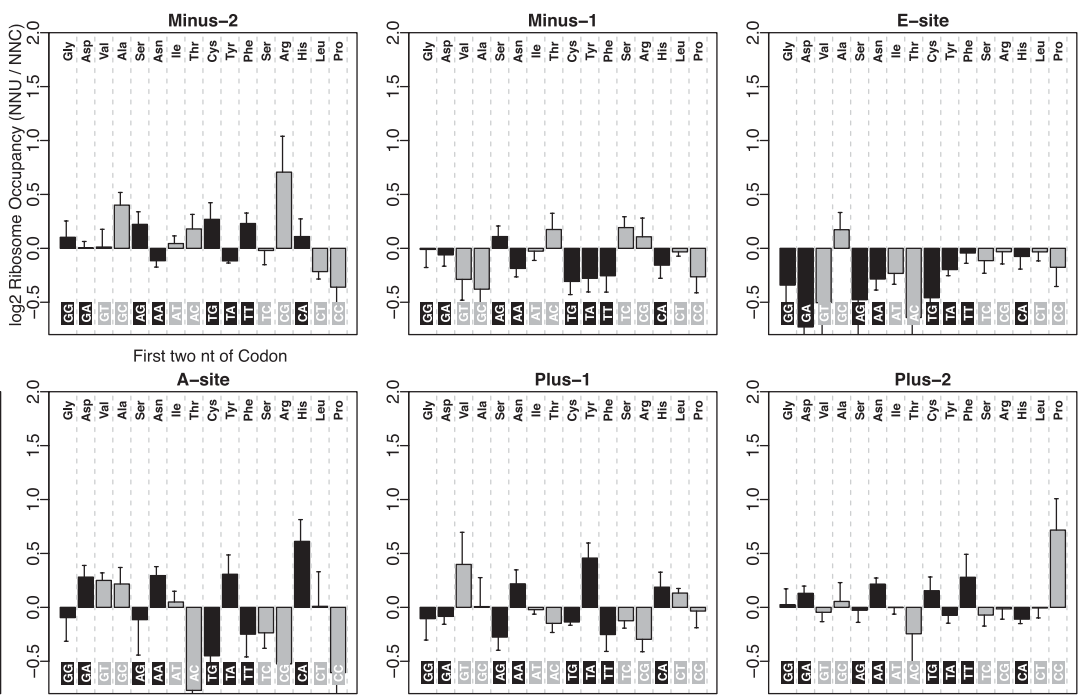

\section{C}
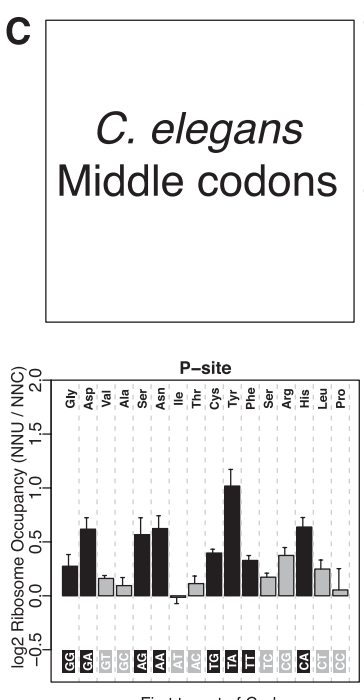

First two nt of Codon

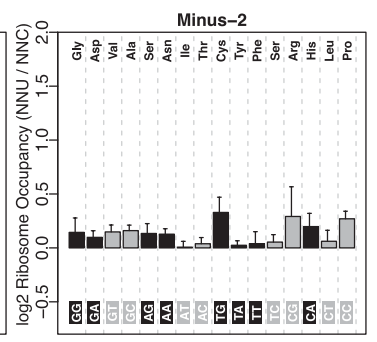

First two nt of Codon

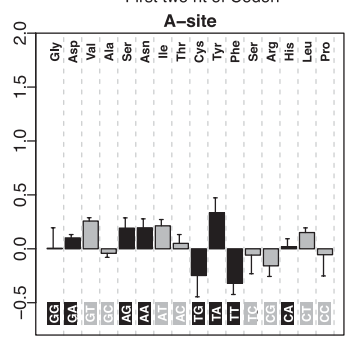

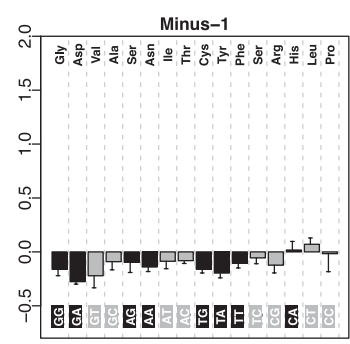
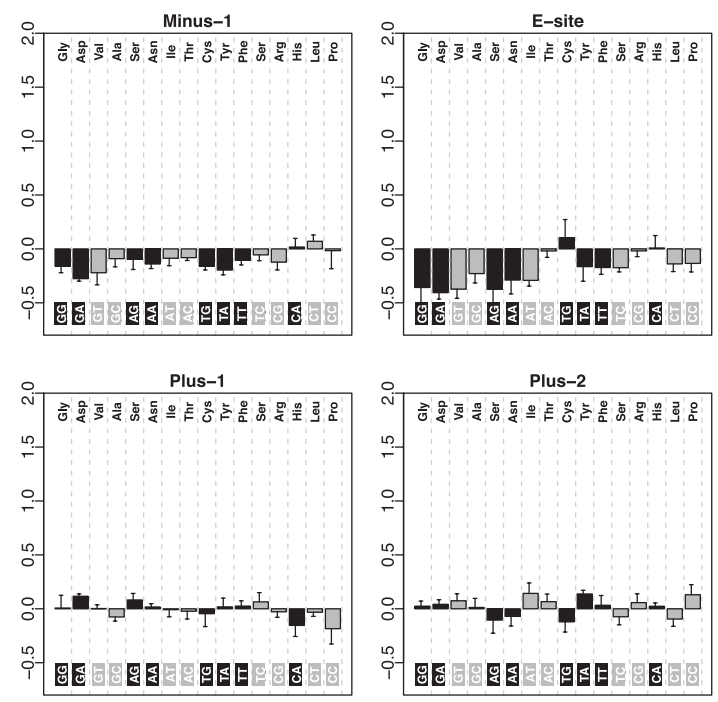

FIGURE 4. Wobble-position ribosome occupancy effects are consistent on different regions of mRNAs in C. elegans. Panels are as in Figure 2. The transcriptome was divided into three sequence sets: $(A)$ the first 50 codons of each message, $(B)$ the last 50 codons, and $(C)$ all coding sequence excluding the first and last 50 codons. Coding sequences that were not long enough to accommodate the partitioning were excluded. Ribosome occupancy calculations were carried out individually on each sequence set identically to calculations shown in Figure 2. 
A

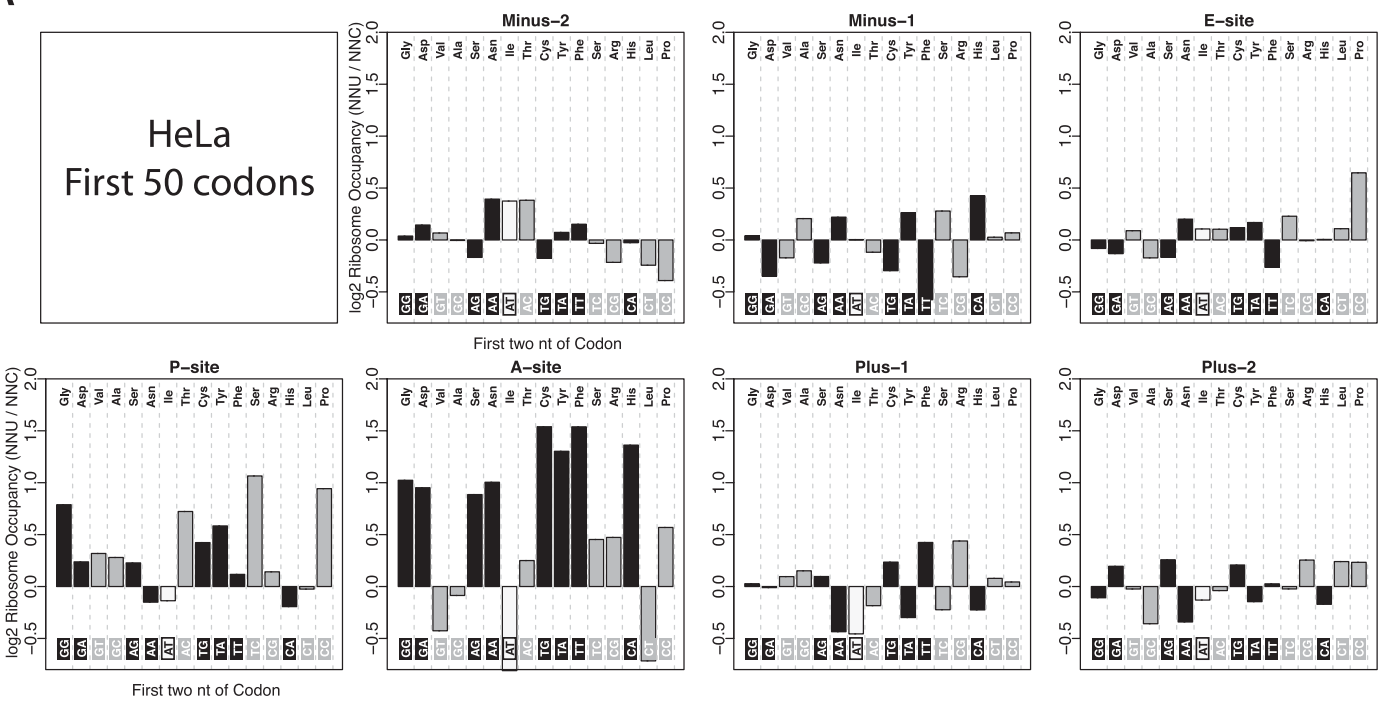

B
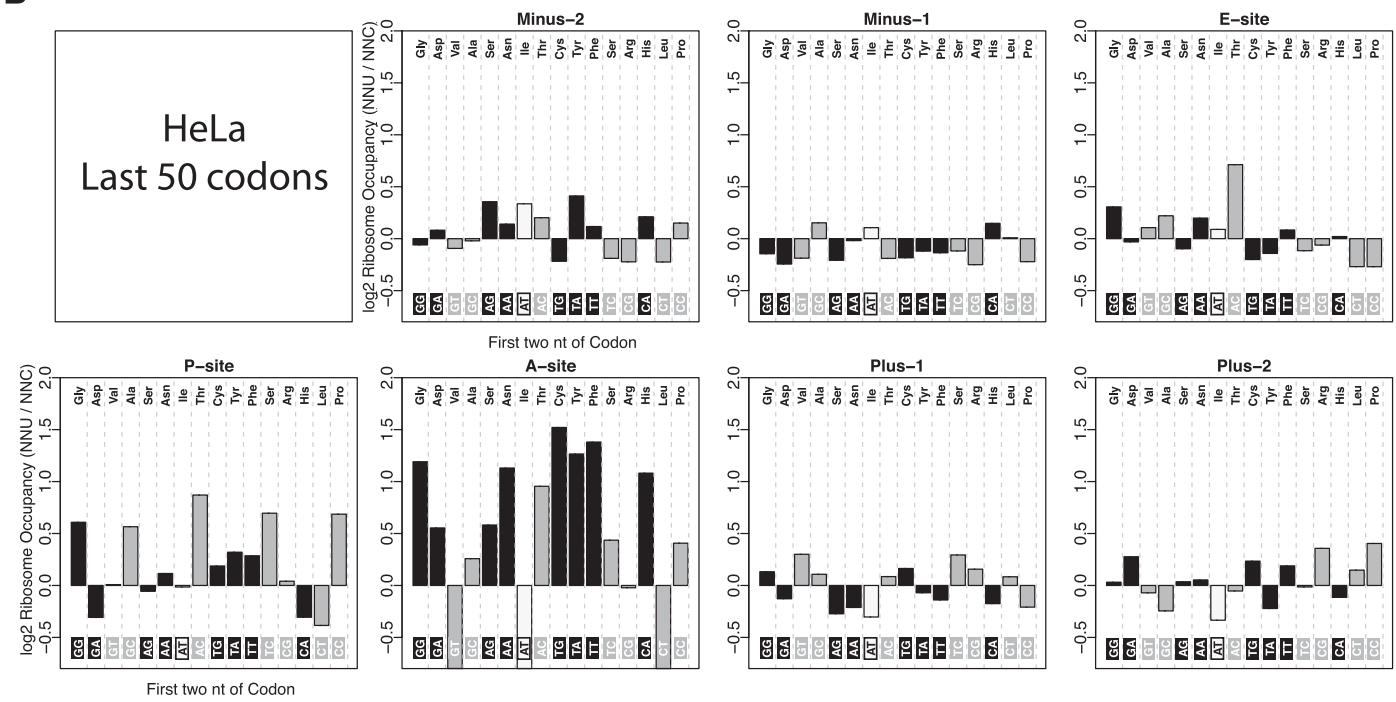

C
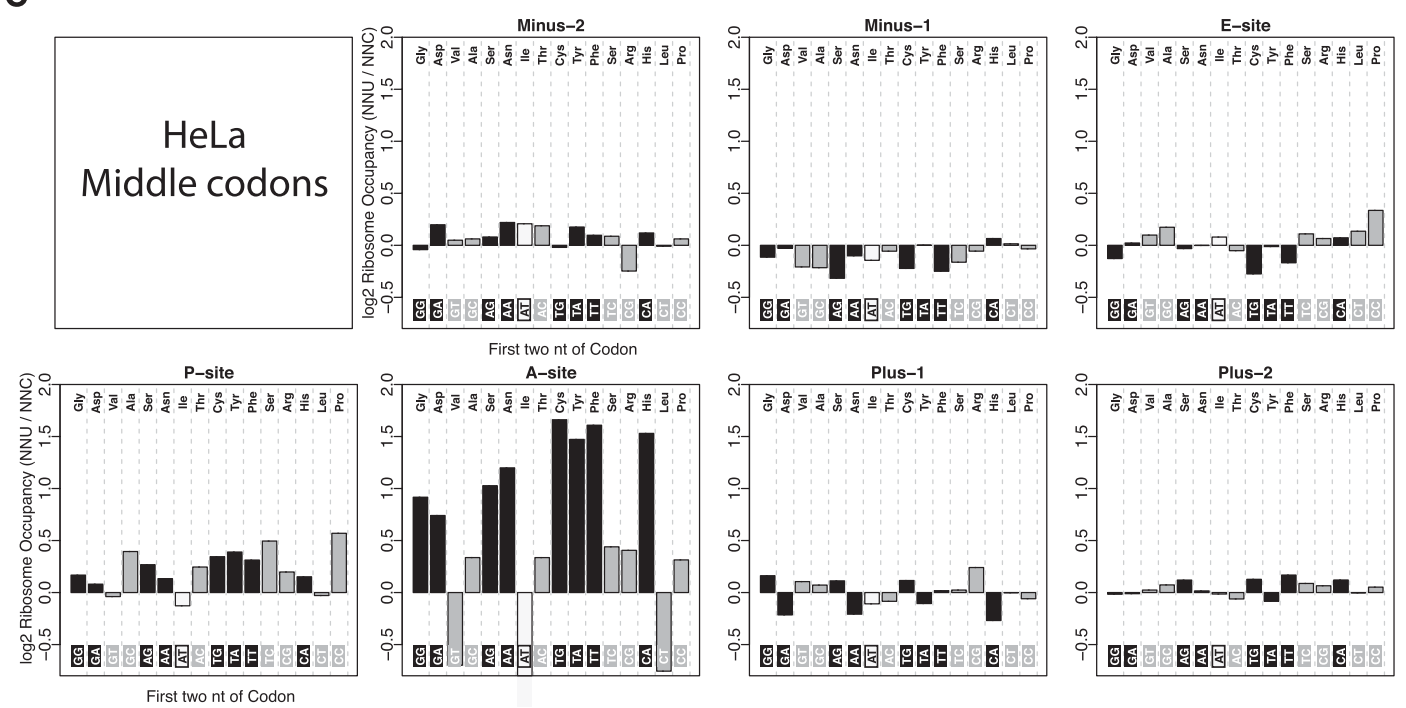

FIGURE 5. Wobble-position ribosome occupancy effects are consistent on different regions of mRNAs in HeLa cells. Panels are as in Figure 2, and data $(A, B, C)$ are derived as described for $C$. elegans in Figure 4. 
effects were consistent with the previous analyses. We also considered that wobble-induced changes in nuclease sensitivity would result in different ribosome occupancy patterns for different sizes of RPF. We repeated the ribosome occupancy analysis individually for specific RPF lengths in the range of 25-35 nt (Supplemental Figs. S4D, S5D), observing consistent wobble-position effects within each individual length class.

Sixth, we consider the possibility that the observed wobble-related effects might reflect ribosome-independent biases in the capture and sequencing of RNA fragments. Certainly there is a precedent for influences of base composition on capture, amplification, and sequencing procedures. In this case, however, it would be very unexpected to have a single internal base (in position 15 of about 30 in C. elegans and 19 of about 32 in HeLa) exhibit a dramatic and individualized effect on capture and sequencing. A number of sources of capture/sequencing bias are additionally ruled out by experiments in which isolated RNA was randomly sheared with alkali and subjected to the same capture and sequencing protocol (Figs. 2B, 3B).

Taken together, these assays support the hypothesis that the enrichment in RPF data sets for fragments containing G:U wobble codons at tRNA-binding sites represents a slowing of translation elongation at such codons, and that this effect is consistent on different classes of mRNA, for wobble codons encoding diverse amino acids, at different stages of ribosome elongation, and in different eukaryotic systems. We propose that the choice of G:U versus G:C base-pairing in codon:anti-codon interactions represents a widely distributed mechanism by which the kinetics of elongation can be fine-tuned in evolution.

It seems likely that such a mechanism might be put to different uses in distinct biological systems. To explore the possibility that "slow" versus "fast" codon choice may have specific roles in individual biological systems (Ran and Higgs 2010), we examined the relationship between codon choice and other aspects of gene activity and function. For $\mathrm{HeLa}$, we observe no association between gene expression levels and a preference between G:C-pairing and wobblepairing codons (Supplemental Fig. S6A,B). In contrast, in C. elegans, we observe that highly expressed genes strongly favor "fast" G:C- and I:C-pairing codons over "slow" G:Uand I:U-pairing codons (Fig. 6A,B). This preference is strongest for $\mathrm{G}: \mathrm{C}$-pairing codons, mirroring the stronger effect of G:C/G:U versus I:C/I:U pairing on ribosome occupancy (Supplemental Fig. S6D). No preference of similar magnitude is observed for third-position nucleotide choice among synonymous NNG/NNA codons (Fig. 6C), nor do intronic regions of highly expressed genes show a preference for $\mathrm{U}$ residues (Supplemental Fig. S6E).

It is likely that expression data from HeLa cells do not accurately identify mRNAs that are abundant across a broad spectrum of human cell-types. However, we expect ribosomal proteins to be one of the most reliably abundant gene
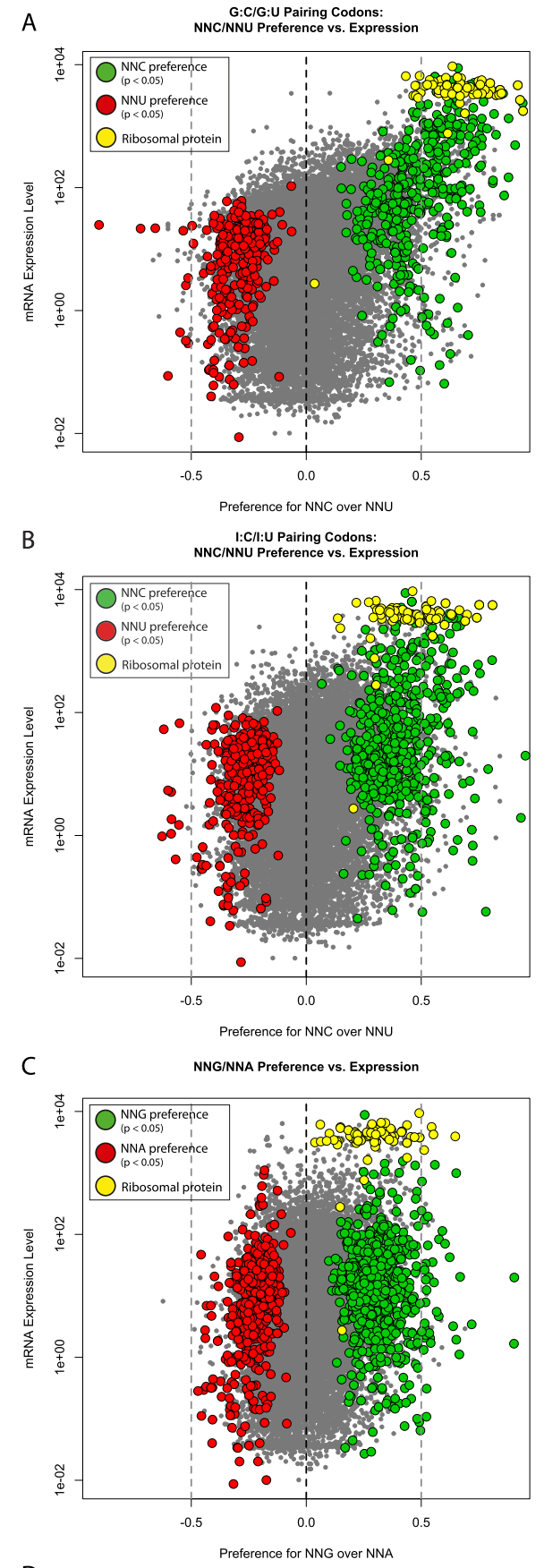

D

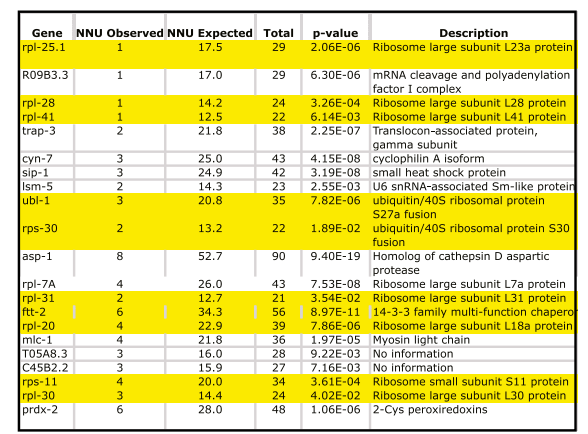

FIGURE 6. (Legend on next page) 
classes across diverse tissues. Indeed, C. elegans ribosomal proteins are among the most highly expressed genes and almost universally show strong preference for G:C- and I:Cpairing codons (Fig. 6A,B). Human ribosomal proteins show no such preference (Supplemental Fig. S6A).

We also considered that human genes' preference for wobble codons may show a correlation with protein levels that is not evident when examining mRNA levels. Cox and Mann (2008) provide quantitation of more than 4000 proteins in HeLa cells. We observed no evident association between wobble codon choice and protein abundance in this data set (Supplemental Fig. S6F-G). Taken together, the data suggest that the preference among highly expressed genes for choosing the "fast" nucleotide at wobble positions may not be conserved between nematodes and mammals.

\section{DISCUSSION}

Position-specific in vivo ribosome elongation rates are certain to be influenced by a variety of factors, including availability of cognate tRNAs, mRNA secondary structure, or the presence of RNA binding proteins. We have here demonstrated one salient feature associated with modulating translation elongation rates: an approximately $15 \%$ to twofold increase in ribosome occupancy at positions where G:U base-pairs are present in the ribosomal decoding center in C. elegans (Supplemental Table S2), and an approximately $65 \%$ to nearly threefold increase in HeLa cells (Supplemental Table S3). These effects are within the normal timescale of translation but are sufficient in magnitude to change the temporal landscape of protein synthesis.

A general ability of wobble codons to slow translational elongation would have potentially interesting mechanistic, regulatory, and evolutionary implications.

On a mechanistic level, increased ribosome occupancy at a codon position on an mRNA could reflect inefficient aminoacyl-tRNA selection (the tRNA being slow to enter the site), slowed translocation (tRNA is slow to leave), or a combination of both. These influences could be focal, affecting only a single chemical step in translation, or could be distributed among several different steps in the mechanism. Although ribosomal footprinting provides remarkable precision in viewing an in vivo process, this precision is not sufficient to determine the individual step(s) affected

FIGURE 6. Highly expressed C. elegans genes avoid slowly translated wobble codons. $(A, B)$ Preference for NNC versus NNU codons for individual genes plotted against expression levels measured by wholeanimal mRNA-seq, averaged across four larval stages. Genes encoding ribosomal proteins are highlighted yellow. Preference is shown separately for codons read by $\mathrm{G}: \mathrm{C} / \mathrm{G}: \mathrm{U}$ at the wobble position $(A)$ and those read by I:C/I:U $(B)$. (C) Preference for NNA versus NNG among synonymous G/A-ending codons plotted against expression level. (D) Table of the most NNC-favoring C. elegans genes from $A$ with preference significant at $P<0.05$ (Bonferroni-corrected). Genes are listed in descending order with the gene showing the greatest preference for NNC codons listed first. by wobble pairing. In particular, the difference that we observe between the two species regarding the site of increased wobble occupancy (the P-site in C. elegans and the A-site in HeLa) may be due to differences in experimental parameters (e.g., a technical difference in kinetics of ribosome freezing in C. elegans sufficient to allow transit of an additional codon) or could reflect differences in the two organisms concerning which biochemical step of translation is retarded by the presence of wobble base-pairs. Discerning the mechanistic details of wobble-induced ribosome slowing is an intriguing topic for future investigation.

At a regulatory/evolutionary level, changes in the temporal landscape of translation could exert a functional influence on biological output in the form of stably and correctly folded protein (Crombie et al. 1992; Zhang et al. 2009, 2010; Siller et al. 2010). One can envision several different means by which coding sequence adjustments could modulate translational rates and, hence, the initial landscapes of protein folding. One such means would be to invoke codons whose translation is limited by paucity of the corresponding tRNA, resulting in translational slowing as the machinery searches for a rare tRNA. In this case, specific tRNA levels would need to be maintained at limiting levels to provide consistent effects on translational rate. As an alternative coding mechanism for modulating translational elongation, wobble-dependent slowing effects would allow fine-tuning of rates in a manner that does not rely on maintenance of borderline tRNA pools. Such a mechanism would allow critical translation rate modulations to be "hard-coded" into mRNA sequences in a proteinspecific way that would be robust to diverse developmental and metabolic conditions.

In addition to the implications for natural regulation of protein synthesis kinetics and quality, our results provide evidence that specific wobble/nonwobble codon choices may influence the biological activity of synthetic genes and genetic constructs used in biotechnology and gene therapy. Indeed optimization of kinetic characteristics - which might require faster or slower peptide synthesis for individual proteins, domains, or residues - could be among the many determinants of effective synthetic biotechnology.

\section{MATERIALS AND METHODS}

For detailed methods, see Supplemental Material.

\section{Tissue}

C. elegans $\mathrm{N} 2$ animals were cultured according the method described by Brenner (1974). HeLa cell monolayers were incubated at $37^{\circ} \mathrm{C}$ in $10 \%$ serum-supplemented Dulbecco modified Eagle medium and washed in phosphate buffered saline. Both HeLa and C. elegans samples were harvested by freezing in liquid nitrogen; these frozen pellets served as the starting material for subsequent manipulations.

Ribosome profiling and sequencing were performed using a protocol very similar to that described by Ingolia et al. (2009), 
with minor modifications described in the Supplemental Methods. Sequencing was performed on an Illumina GAII/GAIIx.

\section{Bioinformatics}

Bioinformatic analyses were carried out using custom Perl and R scripts unless otherwise noted. Sequence reads were mapped using Bowtie v.0.12.2 (Langmead et al. 2009) to the ENSEMBL GRCh37 hg19 gene track for Homo sapiens and the WormBase WS190 (Birney et al. 2004) reference set for C. elegans. In our data analysis, we have used a variety of "stratification" approaches in which subsets of genes or codons are analyzed separately to determine if the different subsets share an observed molecular trend.

\section{Occupancy measurement}

To calculate ribosome occupancy for each codon, we stratified RPF sequences by CU content. First, individual ribosome footprint sequences were assigned to strata defined by C-U differential. Strata with difference values from -5 to 5 were used in this analysis, with analysis carried out in parallel on nonstratified samples giving comparable results. Occupancy calculations were performed independently within each stratum (described below) in order to ensure comparison among sequences of similar composition and remove the influence of composition biases.

Within each CU stratum, we determined a basal "expected" occurrence of each codon $c$ (expected counts $\mathrm{c}_{\mathrm{c}, \mathrm{u}}$ ) by taking the average occurrences of the codon in four "EPA-flanking" sites within the ribosomal footprint (the non-tRNA-interacting sites -2 and -1 to the E-site and +1 and +2 to the A-site).

The representation of each codon $c$ in each stratum $u$ is the ratio of the observed counts of the codon occupying a given ribosomal site, $s$, to the expected counts (determined from the flanking sites):

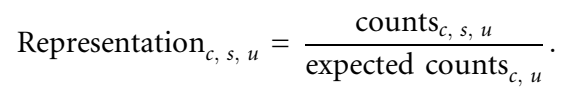

The ribosome occupancy for a given codon-site combination (codon $c$ in residing in site $s$ ) is the average of its representation across $11 \mathrm{CU}$ strata $u$ :

$$
\text { Occupancy }_{c, s}=\frac{\sum_{u=1}^{11} \text { representation }_{c, s, u}}{11} \text {. }
$$

\section{Gene-specific wobble preference}

To assess a gene's preference for using wobble-position G:C versus $\mathrm{G}: \mathrm{U}$ base-pairs, we first calculated the probability of encountering a $\mathrm{G}$ :C for each synonymous $\mathrm{C} / \mathrm{U}$-ending codon pair, $p$, based on the frequency of each codon in the set of all annotated coding sequences for the organism:

$$
P(G: C)_{p}=\frac{\text { Counts }_{N N C, \text { all } C D S}}{\text { Counts }_{N N C, \text { all } C D S}+\text { Counts }_{N N U, \text { all } C D S}} .
$$

From these probabilities, we calculated an expected number of G:C codons expected for each gene, $g$, based on the number of occurrences of each synonymous $\mathrm{C} / \mathrm{U}$-ending codon pair (either $\mathrm{NNC}$ or NNU) and the probability of encountering the G:C codon calculated for each pair in Equation 5:

$$
\text { Expected }(\mathrm{G}: \mathrm{C})=\sum_{\mathrm{p} \in \text { all G:C/G:U pairs }} \text { Count }_{p \text { in } g} \bullet P(G: C)_{p} \text {. }
$$

A gene's preference for $\mathrm{G}: \mathrm{C}$ versus $\mathrm{G}: \mathrm{U}$ wobble pairing is determined by

$$
\text { Preference }(\mathrm{G}: \mathrm{C} \text { to } \mathrm{G}: \mathrm{U})=\frac{\text { Observed }_{G: C}-\text { Expected }_{G: C}}{\text { Expected }_{G: C}} \text {. }
$$

By comparing the number of observed G:C-pairing codons found in a gene's coding sequence to the number expected, we can examine the preference of each gene for $\mathrm{G}: \mathrm{C}$ or $\mathrm{G}: \mathrm{U}$ wobble pairings in a manner that accounts for the relative codon usages of each synonymous pair. An identical analysis was conducted using the set of I:C/I:U-pairing codons.

\section{SUPPLEMENTAL MATERIAL}

Supplemental material is available for this article.

\section{ACKNOWLEDGMENTS}

We thank the following individuals for help and support: E. Tanner, A. Sidow, Z. Weng, P. Lacroute, N. Ingolia, J. Barral, H. Epstein, E. Siller, P. Sarnow, K. Wehner, G. Fuchs, K. Kirkegaard, C. Yanofsky, C. Krishna, J. Maniar, J. Pak, K. Artiles, H. Zhang, S. Gu, P. Parameswaran, L. Gracey, C. Mello, A. Lamm, and D. Wu. This work was supported by grants to A.F. from NIGMS (RO1GM37706), NIAID (U54065359). M.S. was supported by the Stanford Genome Training Program (T32-HD00044), Stanford Graduate Fellowship Program, and a National Science Foundation Graduate Research Fellowship.

Received June 22, 2011; accepted August 30, 2011.

\section{REFERENCES}

Birney E, Andrews T, Bevan P, Caccamo M, Chen Y, Clarke L, Coates G, Cuff J, Curwen V, Cutts T, et al. 2004. An overview of Ensembl. Genome Res 14: 925-928.

Bonekamp F, Dalboge H, Christensen T, Jensen K. 1989. Translation rates of individual codons are not correlated with tRNA abundances or with frequencies of utilization in Escherichia coli. J Bacteriol 171: 5812-5816.

Brenner S. 1974. The genetics of Caenorhabditis elegans. Genetics 77: 71-94.

Chan P, Lowe T. 2008. GtRNAdb: a database of transfer RNA genes detected in genomic sequence. Nucleic Acids Res 37: 93-97.

Cox J, Mann M. 2008. MaxQuant enables high peptide identification rates, individualized p.p.b.-range mass accuracies, and proteomewide protein quantification. Nat Biotechnol 12: 1367-1372.

Crick F. 1966. Codon-anticodon pairing: the wobble hypothesis. J Mol Biol 19: 548-555.

Crombie T, Swaffield J, Brown A. 1992. Protein folding within the cell is influenced by controlled rates of polypeptide elongation. $\mathrm{J} \mathrm{Mol}$ Biol 228: 7-12. 
Curran J, Yarus M. 1989. Rates of aminoacyl-tRNA selection at 29 sense codons in vivo. J Mol Biol 209: 65-77.

Grantham R, Gautier C, Gouy M. 1980. Codon frequencies in 119 individual genes confirm consistent choices of degenerate bases according to genome type. Nucleic Acids Res 8: 1893-1912.

Grantham R, Gautier C, Gouy M, Jacobzone M, Mercier R. 1981. Codon catalog usage is a genome strategy modulated for gene expressivity. Nucleic Acids Res 9: r43-r74.

Ingolia N, Ghaemmaghami S, Newman J, Weissman J. 2009. Genomewide analysis in vivo of translation with nucleotide resolution using ribosome profiling. Science 324: 218-223.

Kato M, Nishikawa K, Uritani M, Miyazaki M, Takemura S. 1990. The difference in the type of codon-anticodon base pairing at the ribosomal P-site is one of the determinants of the translational rate. J Biochem 107: 242-247.

Kim S, Lund J, Kiraly M, Duke K, Jiang M, Stuart J, Eizinger A, Wylie B, Davidson G. 2001. A gene expression map for Caenorhabditis elegans. Science 293: 2087-2092.

Langmead B, Trapnell C, Pop M, Salzberg S. 2009. Ultrafast and memory-efficient alignment of short DNA sequences to the human genome. Genome Biol 10: R25. doi: 10.1186/gb-2009-10-3-r25.

Nakamura M, Sugiura M. 2011. Translation efficiencies of synonymous codons for arginine differ dramatically and are not correlated with codon usage in chloroplasts. Gene 472: 50-54.

Nussinov R. 1981. Eukaryotic dinucleotide preference rules and their implications for degenerate codon usage. J Mol Biol 149: 125131.

Palidwor G, Perkins T, Xia X. 2010. A general model of codon bias due to GC mutational bias. PLoS ONE 5: e13431. doi: 10.1371/ journal.pone.0013431.

Pedersen S. 1984. Escherichia coli ribosomes translate in vivo with variable rate. $E M B O J$ 3: 2895-2898.

Purvis I, Bettany A, Santiago T, Coggins J, Duncan K, Eason R, Brown A. 1987. The efficiency of folding of some proteins is increased by controlled rates of translation in vivo: A hypothesis. J Mol Biol 193: 413-417.

Ran W, Higgs P. 2010. The influence of anticodon-codon interactions and modified bases on codon usage bias in bacteria. Mol Biol Evol 27: 2129-2140.

Siller E, DeZwaan D, Anderson J. 2010. Slowing bacterial translation speed enhances eukaryotic protein folding efficiency. J Mol Biol 396: $1310-1318$.

Sorensen M, Kurland C, Pedersen S. 1989. Codon usage determines translation rate in Escherichia coli. J Mol Biol 207: 365-377.

Steitz J. 1969. Polypeptide chain initiation: nucleotide sequences of the three ribosomal binding sites in bacteriophage R17 RNA. Nature 224: 957-964.

Steitz T. 2008. A structural understanding of the dynamic ribosome machine. Nat Rev Mol Cell Biol 9: 242-253.

Thomas L, Dix D, Thompson R. 1988. Codon choice and gene expression: Synonymous codons differ in their ability to direct aminoacylated-transfer RNA binding to ribosomes in vitro. Proc Natl Acad Sci 85: 4242-4246.

Tuller T, Carmi A, Vestsigian K, Navon S, Dorfan Y, Zaborske J, Pan T, Dahan O, Furman I, Pilpel Y. 2010. An evolutionarily conserved mechanism for controlling the efficiency of protein translation. Cell 141: 344-354.

Varenne S, Buc J, Lloubes R, Lazdunski C. 1984. Translation is a nonuniform process. Effect of tRNA availability on the rate of elongation of nascent polypeptide chains. J Mol Biol 180: 549-576.

Xia X. 1996. Maximizing transcription efficiency causes codon usage bias. Genetics 144: 1309-1320.

Zhang G, Hubalewska M, Ignatova Z. 2009. Transient ribosomal attenuation coordinates protein synthesis and co-translational folding. Nat Struct Mol Biol 16: 274-280.

Zhang F, Saha S, Shabalina S, Kashina A. 2010. Differential arginylation of actin isoforms is regulated by coding-sequence-dependent degradation. Science 329: 1534-1537. 

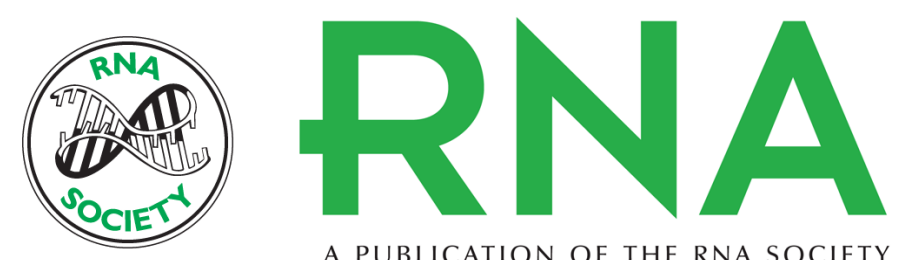

A PUBLICATION OF THE RNA SOCIETY

\section{Wobble base-pairing slows in vivo translation elongation in metazoans}

Michael Stadler and Andrew Fire

RNA 2011 17: 2063-2073 originally published online November 1, 2011

Access the most recent version at doi:10.1261/rna.02890211

Supplemental
Material http://rnajournal.cshlp.org/content/suppl/2011/10/18/rna.02890211.DC1

References This article cites 30 articles, 6 of which can be accessed free at:

http://rnajournal.cshlp.org/content/17/12/2063.full.html\#ref-list-1

\section{License}
Email Alerting Receive free email alerts when new articles cite this article - sign up in the box at the Service top right corner of the article or click here.

